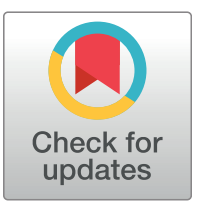

\section{OPEN ACCESS}

Citation: Amer YS, Al-Joudi HF, Varnham JL, Bashiri FA, Hamad MH, Al Salehi SM, et al. (2019)

Appraisal of clinical practice guidelines for the management of attention deficit hyperactivity disorder (ADHD) using the AGREE II Instrument: A systematic review. PLoS ONE 14(7): e0219239. https://doi.org/10.1371/journal.pone.0219239

Editor: Pan Lin, South-Central University for Nationalities, CHINA

Received: July 21, 2018

Accepted: June 19, 2019

Published: July 5, 2019

Copyright: ๑ 2019 Amer et al. This is an open access article distributed under the terms of the Creative Commons Attribution License, which permits unrestricted use, distribution, and reproduction in any medium, provided the original author and source are credited.

Data Availability Statement: All relevant data are within the paper and its Supporting Information files.

Funding: This work was supported by Saudi ADHD Society. URL: https://adhd.org.sa/. This is part of a comprehensive project for adaptation of National CPG for ADHD sponsored by the Saudi ADHD Society. The funders had no role in study design, data collection and analysis, decision to publish, or preparation of the manuscript.

RESEARCH ARTICLE

\title{
Appraisal of clinical practice guidelines for the management of attention deficit hyperactivity disorder (ADHD) using the AGREE II Instrument: A systematic review
}

\begin{abstract}
Yasser Sami Amer ${ }^{1,2,3,4 *}$, Haya Faisal Al-Joudi ${ }^{5}$, Jeremy L. Varnham ${ }^{6,7}$, Fahad A. Bashiri ${ }^{8}$, Muddathir Hamad Hamad ${ }^{8}$, Saleh M. Al Salehi ${ }^{9}$, Hadeel Fakhri Daghash ${ }^{\mathbf{1 0}}$, Turki Homod Albatti $^{6,11}$, on behalf of The Saudi ADHD Society ${ }^{\text {I }}$

1 CPG Unit, Quality Management Department, King Saud University Medical City, Riyadh, Saudi Arabia, 2 Pediatrics Department, King Khalid University Hospital, King Saud University Medical City, Riyadh, Saudi Arabia, 3 Research Chair for Evidence-Based Health Care and Knowledge Translation, Deanship of Scientific Research, King Saud University, Riyadh, Saudi Arabia, 4 Alexandria Center for Evidence-Based Clinical Practice Guidelines, Alexandria University, Alexandria, Egypt, 5 Department of Neurosciences, King Faisal Specialist Hospital and Research Center, Riyadh, Saudi Arabia, 6 Saudi ADHD Society, Riyadh, Saudi Arabia, 7 School of Psychology, University of East London, London, United Kingdom, 8 Division of Neurology, Department of Pediatrics, College of Medicine, King Saud University Medical City, King Saud University, Riyadh, Saudi Arabia, 9 Child Development Center, King Abdullah Bin Abdulaziz University Hospital, Princess Noura Bint AbdulRahman University, Riyadh, Saudi Arabia, 10 Ada'a Program, Assistant Deputyship for Hospital Services, Ministry of Health, Riyadh, Saudi Arabia, 11 Department of Psychiatry, King Saud University Medical City, King Saud University, Riyadh, Saudi Arabia
\end{abstract}

II Membership of the Saudi ADHD Society is provided in the Acknowledgments

*yassersamiamer@gmail.com

\section{Abstract}

\section{Background and objective}

High quality evidence-based clinical practice guidelines (CPGs) have a major impact on the appropriate diagnosis and management and positive outcomes. The evidence-based healthcare for patients with attention deficit hyperactive disorder (ADHD) is challenging. The objective of this study was to appraise the quality of published CPGs for ADHD.

\section{Methods}

A systematic review was conducted for ADHD CPGs using CPG databases, DynaMed, PubMed, and Google Scholar. The quality of each included CPG was appraised by three independent appraisers using the Appraisal of Guidelines for Research \& Evaluation II (AGREE II) instrument.

\section{Results}

Six CPGs were critically reviewed. The AGREE II standardized domain scores revealed variation between the quality of these CPGs with the National Institute of Health and Care Excellence (NICE), University of Michigan Health System, and American Academy of 
Competing interests: The authors have declared that no competing interests exist.
Pediatrics CPGs as the top three. Overall, the recommendations for management of ADHD were similar in these CPGs.

\section{Conclusions}

Reporting of CPG development is often poorly documented. Guideline development groups should aim to follow the AGREE II criteria to improve the standards and quality of CPGs. The NICE CPG showed the best quality. Embedding the AGREE II appraisal of CPGs in the training and education of healthcare providers is recommended.

The protocol for this study was published in PROSPERO (International prospective register of systematic reviews). Link: http://www.crd.york.ac.uk/PROSPERO/display_record. php?|D=CRD42017078712 and is additionally available from protocols.io. Link: https://dx. doi.org/10.17504/protocols.io.q27dyhn.

\section{Introduction}

Attention deficit hyperactivity disorder [1,2] or Attention-Deficit/ Hyperactivity Disorder $[3,4](\mathrm{ADHD})$, is a chronic neurodevelopmental disorder characterized by developmentally inappropriate levels of hyperactivity-impulsivity and/or inattention [1-9]. ADHD is clinically and genetically heterogeneous with multiple possible etiologies and frequent neuropsychiatric comorbidities [10,11]. ADHD is highly prevalent in $5-6 \%$ of children and in $3.8-4.4 \%$ of adults [12].

Clinical practice guidelines (CPGs) summarize the best available evidence and provide guidance for healthcare providers during their daily practice. CPGs can support the knowledge-to-action cycle effectively if they were developed using a systematic and rigorous methodology. Published evidence has revealed that CPGs can improve patient outcomes, patient experience, and quality and safety in healthcare [13].

In 2011, the Health and Medicine Division (HMD) of the American National Academies, formerly the Institute of Medicine (IOM), published its eight criteria of trustworthy CPGs, Clinical Practice Guidelines We Can Trust [14]. Since then, many sets of standards or criteria for high quality CPGs have been published or updated, including the Guidelines International Network's [15], the GIN-McMaster Checklist [16], and the AGREE II Reporting Checklist [17], based upon the AGREE II Instrument's 23 criteria. These standards have helped in shaping the development process and methodologies of CPGs worldwide [18].

Two systematic reviews of CPG appraisal tools have included a total of 64 tools [19,20]; these revealed that the AGREE II Instrument was the only tool that had a validated scoring system, as well as already being widely adopted. It has proven to become the international gold standard for quality assessment and development of CPGs, being cited more than 746 times between 2013-2018 [21].

A brief review of literature on the utilization of AGREE II for ADHD CPGs revealed two uses: One was restricted to psychopharmacological management of ADHD [22], and the other was conducted as part of a Master's thesis in Pediatrics at Alexandria University [23]. The primary objective of this study is to provide a comprehensive, easily accessible, and updated assessment of the quality of available CPGs pertaining to ADHD diagnosis and management, using the gold standard instrument, AGREE II; CPGs included were published between 2012 and 2019, following the publication of the HDM and G-I-N CPG standards. Earlier published 
CPGs in general were found to be of variable quality and poor compliance with available methodological standards at that time $[24,25]$

\section{Methods}

The protocol for this study was published in PROSPERO (International prospective register of systematic reviews). Link: http://www.crd.york.ac.uk/PROSPERO/display_record.php?ID= CRD42017078712 [26] and is also available from Protocols.io. Link: https://dx.doi.org/10. 17504/protocols.io.q27dyhn [25].

\section{Eligibility criteria}

Criteria for including CPGs were: (1) Evidence-based CPGs (i.e. with a clear description of the development methodology); (2) English language; (3) original source CPGs (de novo developed); (4) both national and international CPGs; (5) published between January 1, 2012 and July 1, 2017 (the search was further extended till June 15, 2019); (6) published by an organization or group authorship in a CPG database or peer-reviewed journal. Only the most current version of each source CPG was included whether in the format of a full CPG document retrieved from the developing organization's official website or in the form of a full-text publication that was authored by the CPG development group.

We excluded CPGs that were published earlier than 2012, written in non-English language, presented as consensus or expert-based statements or CPGs, adapted from other source CPG (s), or that had single authorship. Relevant publications summarizing or reporting implementation of the included CPGs by different authors were not considered for this CPG appraisal.

\section{Information resources (identification of ADHD CPGs)}

We used literature searches of bibliographic databases (Medline/PubMed and Google Scholar), EBSCO DynaMed Plus (US), and CPG databases: American Agency for Healthcare Research and Quality's (AHRQ) National Guideline Clearinghouse (US), Guidelines International Network (GIN), Scottish Intercollegiate Guidelines Network (SIGN; UK), National Institute of Health and Care Excellence (NICE; UK), and the Australian National Health and Medical Research Council (NHMRC). We also searched databases of national and international societies specializing in fields related to ADHD (e.g. American Psychiatric Association, European Psychiatric Association).

\section{Search, Screen, and Study Selection}

Keywords used included "attention-deficit/hyperactivity disorder" or "ADHD," and "guideline," "practice guideline," "clinical practice guideline," "practice parameter," "guidance," or "recommendations" [26-28].

The PubMed search strategy included "attention deficit hyperactivity disorder"[tiab] OR "ADHD"[tiab]. Filters activated: Guideline, Publication date from 2012/01/01 to 2018/06/30 (extended to 2019/6/15), Humans.

Additionally, we used the PIPOH (Patient Population, Interventions, Professionals, Outcomes, and Healthcare Setting) checklist $[18,28]$ to further assist in the inclusion and exclusion process. The following is a description of the characteristics derived from and used via PIPOH: Our patient population $(\mathrm{P})$ was children and adults being assessed for or with a diagnosis of ADHD. Interventions (I) included diagnosis (complaints of the parent, teacher, or adolescent, history and physical examination, psychological tools, investigations, comorbidities) and treatment (pharmacological treatment, psychological and behavioral interventions, adverse effects 
of treatments, treatment of adverse effects, monitoring, special cases, complementary medicine, and transition of care from childhood to adulthood). Type of professionals (P) included physicians (e.g. psychiatrists, pediatricians, and neurologists), clinical psychologists, pharmacists, nurses, dieticians, occupational therapists, and social workers. Major outcomes $(\mathrm{O})$ included ADHD symptom severity, academic performance, functional status, side effects of stimulant medications, and quality of life. Healthcare setting $(\mathrm{H})$ included primary, secondary, and tertiary care settings addressing assessment, treatment, and management of ADHD.

Two reviewers (YA, JV) independently screened titles and abstracts of retrieved CPGs and articles meeting the inclusion criteria. The screening was rechecked by three other reviewers (TA, FB, MH). Disagreements were resolved by further discussions with the entire group after retrieval and review of the full CPG documents or full-text articles, including links to any available supplemental documents or web pages. We repeated our search before the final manuscript re-submission in June 2019 based on the pre-publication peer review to identify any new eligible CPGs.

\section{Assessment of CPGs using the AGREE II Instrument}

The AGREE II Instrument (www.agreetrust.org) consists of 23 items or questions organized in six domains including scope and purpose (items 1-3), stakeholder involvement (items 4-6), rigor of development (items 7-14), clarity of presentation (items 15-17), applicability (items 18-21), and editorial independence (items 22-23). Each item or question is scored on a Likert scale from one to seven, where $1=$ strongly disagree and $7=$ strongly agree. The AGREE II assessment was conducted by using the "My AGREE PLUS" online tool developed by the AGREE Enterprise. My AGREE PLUS supports the AGREE II assessment process by creating a CPG "appraisal group" for each CPG, compiling and calculating the items' scores into domain scores, and generating the final reports. My AGREE PLUS users are required to complete a free registration process before starting the AGREE online assessment for a given CPG. Each CPG appraisal group is handled by a "coordinator" who registers group's details, invites assessors, reviews data, and generates the final AGREE II reports. Two separate reports can be generated from My AGREE PLUS once the CPG group assessment is completed: One for the "ratings" (i.e. individual item scores and standardized domain scores) and another for the "comments." Additionally, the AGREE website provides online audiovisual training resources for using the AGREE II Instrument, as well as videos describing different functionalities of the My AGREE PLUS online platform.

Seven AGREE II assessors were selected with a wide range of clinical expertise (a child psychiatrist, two pediatric neurologists, a developmental pediatrician, a clinical neuropsychologist, a clinical pharmacist, and a general pediatrician and CPGs methodologist). At the outset of this project, AGREE II capacity building was conducted for the assessors by the expert methodologist through training and hands-on sessions in the concepts and standards of CPGs, and using the instrument. Each reviewer independently scored his/her assigned CPGs. Each one of the included CPGs was independently appraised by three reviewers: two clinicians and a methodologist.

All assessors reviewed the full CPG document, in addition to any supplementary documents or links to online pages related to the guideline's methodology or implementation tools. For each item, AGREE assessors were asked to record the rationale for their scores in the comment section. Differences between assessors' scores were resolved by asking those who had provided outlying scores to re-assess after discussion with the group. The disagreements were mainly observed in questions highly related to the CPG development methodology (i.e. questions 7-14 of domain 3) and implementation (especially question 18 of domain 5). The 
percentage of preliminary disagreements in some CPGs was 9 per 23 questions (39\%) but were less in subsequently appraised CPGs with the rising learning and understanding curve for utilizing the AGREE II criteria for quality assessment. The standardized AGREE domain scores (ranging from 0 to 100\%) were automatically calculated by My AGREE PLUS following the equations provided by the AGREE II User's Manual.

A cut-off point of $60 \%$ for each AGREE standardized domain score was agreed upon by the reviewers, with more weight emphasized on the scores of domains three and five to facilitate the final assessment of the reporting quality of CPGs. Similar categorization of domains was recently reported and published $[13,29,30]$.

An additional validation of the six CPGs for inclusion of systematic reviews with or without meta-analyses in their evidence-base and the frequency and percentage of Cochrane systematic reviews among these reviews was conducted.

Moreover, we checked whether the Grading of Recommendations Assessment, Development and Evaluation (short GRADE) methodology was utilized for the CPG development process as several CPG developing organizations are increasingly shifting to using the GRADE (e.g. World Health Organization, NICE, SIGN, NHMRC, etc.) [31-34]. The GRADE is a method of assessing the certainty in evidence (i.e. quality of evidence or confidence in effect estimates) and the strength of recommendations in health care. It has important implications for summarizing evidence for systematic reviews, health technology assessments, and CPGs as well as other decision makers $[35,36]$.

\section{Results}

\section{Identification of ADHD CPGs}

The results of the search were summarized in S1 Fig. The initial list of 30 retrieved CPGs was reviewed, discussed and filtered by the assessors. Six recent ADHD CPGs complying with the identified PIPOH and inclusion criteria were eligible. These CPGs were developed by the American Academy of Pediatrics (AAP) in 2012, the University of Michigan Health System (UMHS) in 2012, the National Institute of Health and Care Excellence (NICE) in 2016 (updated in 2018), the National Health Medical Research Center (NHMRC) in 2013, the Canadian ADHD Resource Alliance (CADDRA) in 2014 (updated in 2018), and the Singapore Ministry of Health (SMOH) in 2014 [37-42].

An updated search and screen was conducted for ADHD Source CPGs in June 2019 using the same aforementioned information resources and criteria. This repeated search did not reveal any eligible CPG that needed to be added to the previous AGREE appraisal. Though excluded, several recent CPGs or relevant online material were worthwhile to mention due to the national and/or international impact of their publishing organizations.

Examples of these (with reasons for exclusion) include (i) the Interdisciplinary Evidenceand Consensus-based Guideline "ADHD in Children, Young People and Adults" June 2018 by the Association of the Scientific Medical Societies in Germany (AWMF) Online (in German) [43]; (ii) CPG on Therapeutic Interventions in ADHD 2017 by the Working group of the Clinical Practice Guideline on Therapeutic Interventions in ADHD, Ministry of Health, Social Services and Equality, Health Sciences Institute in Aragon (IACS) (in Spanish) [44]; (iii) the Updated European Consensus Statement on diagnosis and treatment of adult ADHD 2019 by the European Network Adult ADHD (ENAA) (Consensus statement) [45], (iv) British Association for Psychopharmacology's (BAP) CPG for the pharmacological management of ADHD 2014 (Consensus statement) [46], and (v) the Centers for Disease Control and Prevention endorsed and posted the AAP treatment recommendations in their official website as of September 2018 (adopted AAP 2011 CPG) [47]. 
Table 1. Characteristics of the ADHD clinical practice guidelines (general).

\begin{tabular}{|c|c|c|c|c|c|}
\hline Title & $\begin{array}{c}\text { Year of } \\
\text { publication }\end{array}$ & Country & $\begin{array}{c}\text { Level of } \\
\text { development }\end{array}$ & $\begin{array}{l}\text { Organization } \\
\text { (short name) }\end{array}$ & $\begin{array}{l}\text { Total number of } \\
\text { references }\end{array}$ \\
\hline $\begin{array}{l}\text { American Academy of Pediatrics (AAP) CPG on diagnosis, evaluation, } \\
\text { and treatment of ADHD in children and adolescents (37) }\end{array}$ & 2012 & $\begin{array}{l}\text { United } \\
\text { States }\end{array}$ & National & AAP & 70 \\
\hline $\begin{array}{l}\text { Canadian ADHD Resource Alliance (CADDRA)-Canadian ADHD CPGs } \\
\text { (CAP-Guidelines) } 3^{\text {rd }} \text { Edition (updated in } 4^{\text {th }} \text { edition). ( } 39 \text { ) }\end{array}$ & $\begin{array}{l}2015 \text { (updated } \\
\text { 2018) }\end{array}$ & Canada & National & CADDRA & 264 \\
\hline $\begin{array}{l}\text { National Health and Medical Research Council (NHMRC) Clinical } \\
\text { Practice Points on diagnosis, assessment, and management of ADHD in } \\
\text { children and adolescents (40) }\end{array}$ & 2014 & Australia & National & NHMRC & 112 \\
\hline $\begin{array}{l}\text { National Institute for Health and Care Excellence (NICE) ADHD CPG } \\
(42)\end{array}$ & $\begin{array}{l}2016 \text { (updated } \\
\text { 2018) }\end{array}$ & $\begin{array}{l}\text { United } \\
\text { Kingdom }\end{array}$ & National & NICE & 2941 \\
\hline Singapore Ministry of Health (SMOH) Guideline on ADHD (41) & 2013 & Singapore & National & $\mathrm{SMOH}$ & 250 \\
\hline University of Michigan Health System ADHD (UMHS) (38) & 2013 & USA & Local & UMHS & 14 \\
\hline
\end{tabular}

https://doi.org/10.1371/journal.pone.0219239.t001

A number of online resources for the professionals and public summarized and discussed the recommendations of some these CPGs and relevant documents for example DynaMed Plus (updated in May 2019) [48,49], the World Federation ADHD Guide (2019) [50], and the ADHD Institute (updated in March 2019) [51] but without using a formal CPG appraisal tool like the AGREE II Instrument [19,20].

\section{Key characteristics of CPGs}

Tables 1 and 2 demonstrate characteristics of all eligible CPGs. The eligible CPGs included five national CPGs (AAP, NICE, NHMRC, CADDRA, and SMOH) and one local CPG (UMHS). Two CPGs were developed by US-based organizations $(n=2,33 \%)$, followed by one CPG $(\mathrm{n}=1,17 \%)$ developed by each of a Canadian-based, UK-based, Australian-based, and Singaporean-based organization respectively. The six included CPGs were developed by governmental bodies $(n=3,50 \%)$, medical specialty society $(n=2,33 \%)$, and healthcare improvement and CPG developer organizations $(n=2,33 \%)$.

Table 2. Characteristics of the ADHD clinical practice guidelines (clinical content/ options of care).

Clinical content/ Options of care

\begin{tabular}{l|c|c|}
\hline & (37) & (39) \\
\hline 1. Parent/ Teacher/ Patient (adolescent) complaints & \multicolumn{2}{c}{ Diagnosis and Assess } \\
\hline 2. Clinical picture & $\mathrm{Y}$ & $\mathrm{Y}$ \\
\hline 3. Psychological Tools & $\mathrm{Y}$ & \\
\hline 4. Differential diagnosis & $\mathrm{Y}$ & \\
\hline 5. Investigations & $\mathrm{N}$ & \\
\hline
\end{tabular}

NHMRC (40)
NICE

(42)
SMOH

(41)
UMHS

(38)

6. Treatment

7. Psychological and Behavioral interventions (PBI)

8. Pharmacological treatment

9. Treatment of adverse effects of pharmacological treatment

10. Comorbidities

11. Monitoring

12. Special cases

13. Complementary medicine

14. Transition of care from childhood to adulthood

\begin{tabular}{|c|c|c|}
\hline Y & Y & \\
\hline N & Y & \\
\hline Y & N & \\
\hline Y & N & \\
\hline Y & N & \\
\hline
\end{tabular}

\begin{tabular}{|c|l|}
\hline $\mathrm{Y}$ & \\
\hline $\mathrm{Y}$ & \\
\hline $\mathrm{N}$ & \\
$\mathrm{Y}$ & \\
\hline $\mathrm{N}$ & \\
\hline
\end{tabular}

\begin{tabular}{|c|c|}
\hline $\mathrm{Y}$ & $\mathrm{Y}$ \\
$\mathrm{N}$ & $\mathrm{Y}$ \\
\hline $\mathrm{N}$ & $\mathrm{Y}$ \\
\hline $\mathrm{Y}$ & $\mathrm{Y}$ \\
\hline $\mathrm{Y}$ & $\mathrm{Y}$ \\
\hline
\end{tabular}

\begin{tabular}{c|c|c|c|c|c}
\multicolumn{7}{c|}{$\mathrm{Y}$} & $\mathrm{Y}$ & $\mathrm{Y}$ & $\mathrm{Y}$ & $\mathrm{Y}$ \\
\hline $\mathrm{Y}$ & $\mathrm{Y}$ & $\mathrm{Y}$ & $\mathrm{Y}$ & $\mathrm{Y}$ & $\mathrm{Y}$ \\
\hline $\mathrm{Y}$ & $\mathrm{Y}$ & $\mathrm{Y}$ & $\mathrm{Y}$ & $\mathrm{N}$ & $\mathrm{Y}$ \\
\hline $\mathrm{Y}$ & $\mathrm{Y}$ & $\mathrm{Y}$ & $\mathrm{Y}$ & $\mathrm{Y}$ & $\mathrm{Y}$ \\
\hline $\mathrm{Y}$ & $\mathrm{Y}$ & $\mathrm{Y}$ & $\mathrm{Y}$ & $\mathrm{Y}$ & $\mathrm{Y}$ \\
\hline $\mathrm{Y}$ & $\mathrm{N}$ & $\mathrm{N}$ & $\mathrm{N}$ & $\mathrm{N}$ & $\mathrm{Y}$ \\
\hline $\mathrm{Y}$ & $\mathrm{N}$ & $\mathrm{Y}$ & $\mathrm{N}$ & $\mathrm{Y}$ & $\mathrm{Y}$ \\
$\mathrm{Y}$ & $\mathrm{N}$ & $\mathrm{Y}$ & $\mathrm{Y}$ & $\mathrm{Y}$ & $\mathrm{N}$ \\
\hline
\end{tabular}

$\mathrm{Y}=$ Yes (included); $\mathrm{N}=$ No (not included) 


\section{Reporting the quality of ADHD CPGs}

The AGREE II standardized domain scores were summarized in Table 3.

Domain 1: Scope and purpose. The AGREE II standardized score for domain 1 ranged from $37 \%$ to $100 \%$. Scores of all CPGs were greater than $60 \%$ in domain 1 except the SMOH CPG, in which the limited description of overall objectives, health questions, and patient populations resulted in a lower score. The two CPGs scoring more than $90 \%$ were NICE and UMHS.

Domain 2: Stakeholder involvement. The AGREE II standardized domain scores for domain 2 ranged from $43 \%$ to $96 \%$. Scores of all CPGs were greater than $60 \%$ in domain 1 except the SMOH, CADDRA, and UMHS CPGs. The lack of adequate descriptions of patient preferences or target users resulted in the low scores for these CPGs. Only the NICE CPG scored more than $90 \%$.

Domain 3: Rigor of development. The AGREE II standardized scores for domain 3 ranged from $35 \%$ to $93 \%$. Three CPGs received scores greater than or equal to $60 \%$ : NICE (93\%),

Table 3. AGREE II domain-standardized scores (ratings) for CPGs on management of ADHD.

\begin{tabular}{|c|c|c|c|c|c|c|}
\hline $\begin{array}{l}\text { ADHD CPGs (reference)/ AGREE II } \\
\text { Domains-standardized scores }\end{array}$ & $\begin{array}{r}\text { AAP } \\
(37)\end{array}$ & $\begin{array}{l}\text { CADDRA } \\
(39)\end{array}$ & $\begin{array}{c}\text { NICE } \\
(42)\end{array}$ & $\begin{array}{c}\text { NHMRC } \\
(40)\end{array}$ & $\begin{array}{c}\text { SMOH } \\
(41)\end{array}$ & $\begin{array}{l}\text { UMHS } \\
(38)\end{array}$ \\
\hline $\begin{array}{l}\text { Domain 1. Scope and Purpose } \\
\text { Items 1-3: Objectives; Health question(s); } \\
\text { Population (patients, public, etc.). }\end{array}$ & $80 \%$ & $74 \%$ & $100 \%$ & $72 \%$ & $37 \%$ & $91 \%$ \\
\hline $\begin{array}{l}\text { Domain 2. Stakeholder Involvement } \\
\text { Items 4-6: Group Membership; Target } \\
\text { population preferences and views; Target } \\
\text { users. }\end{array}$ & $67 \%$ & $50 \%$ & $96 \%$ & $76 \%$ & $59 \%$ & $43 \%$ \\
\hline $\begin{array}{l}\text { Domain 3. Rigour of development } \\
\text { Items 7-14: Search methods; Evidence } \\
\text { selection criteria; Strengths and limitations } \\
\text { of the evidence; Formulation of } \\
\text { recommendations; Consideration of benefits } \\
\text { and harms; Link between recommendations } \\
\text { and evidence; External review; Updating } \\
\text { procedure. }\end{array}$ & $66 \%$ & $35 \%$ & $93 \%$ & $53 \%$ & $47 \%$ & $60 \%$ \\
\hline $\begin{array}{l}\text { Domain 4. Clarity and presentation } \\
\text { Items 15-17: Specific and unambiguous } \\
\text { recommendations; Management options; } \\
\text { Identifiable key recommendations }\end{array}$ & $76 \%$ & $63 \%$ & $89 \%$ & $65 \%$ & $83 \%$ & $81 \%$ \\
\hline $\begin{array}{l}\text { Domain 5. Applicability } \\
\text { Items 18-21: Facilitators and barriers to } \\
\text { application; Implementation advice/ tools; } \\
\text { Resource implications; Monitoring/ auditing } \\
\text { criteria }\end{array}$ & $64 \%$ & $35 \%$ & $92 \%$ & $29 \%$ & $69 \%$ & $69 \%$ \\
\hline $\begin{array}{l}\text { Domain 6. Editorial independence } \\
\text { Items 22, 23: Funding body; Competing } \\
\text { interests }\end{array}$ & $75 \%$ & $78 \%$ & $92 \%$ & $67 \%$ & $28 \%$ & $69 \%$ \\
\hline $\begin{array}{l}\text { Overall Assessment } 1 \\
\text { (Overall quality) }\end{array}$ & $56 \%$ & $67 \%$ & $100 \%$ & $56 \%$ & $50 \%$ & $72 \%$ \\
\hline $\begin{array}{l}\text { Overall Assessment } 2 \\
\text { (Recommend the CPG for use) }\end{array}$ & $\begin{array}{l}\text { Yes-1, Yes with } \\
\text { modifications-2, } \\
\text { No-0 }\end{array}$ & $\begin{array}{l}\text { Yes-1, Yes with } \\
\text { modifications-2, } \\
\text { No-0 }\end{array}$ & $\begin{array}{l}\text { Yes-1, Yes with } \\
\text { modifications-2, } \\
\text { No-0 }\end{array}$ & $\begin{array}{l}\text { Yes-0, Yes with } \\
\text { modifications-3, } \\
\text { No-0 }\end{array}$ & $\begin{array}{l}\text { Yes-0, Yes with } \\
\text { modifications-2, } \\
\text { No-1 }\end{array}$ & $\begin{array}{l}\text { Yes-2, Yes with } \\
\text { modifications-1, } \\
\text { No-0 }\end{array}$ \\
\hline AGREE II Assessors & $H A, T A, Y A$ & $S A, T A, Y A$ & $F B, M H, Y A$ & $H D, T A, Y A$ & $F B, H D, Y A$ & $H A, S A, Y A$ \\
\hline
\end{tabular}

American Academy of Pediatrics (AAP), the University of Michigan Health System (UMHS), National Institute of Health and Care Excellence (NICE), the National Health Medical Research Center (NHMRC), the Canadian ADHD Resource Alliance (CADDRA), and the Singapore Ministry of Health (SMOH), Clinical Practice Guidelines (CPGs), AGREE II (Appraisal of Guidelines for Research and Evaluation Instrument Version II) 
AAP (66\%), and UMHS (60\%). The rest (NHMRC, CADDRA, and SMOH) received less than $60 \%$ in domain 3. Comprehensive search methods and strategy, evidence selection criteria, strengths and limitations of the evidence (evidence tables), detailed process of formulation of recommendations, discussion of the process of trade-off between risks and benefits, process of external review, and details of the updating process were the most common weaknesses among the NHMRC, CADDRA, and SMOH CPGs.

Domain 4: Clarity of presentation. The AGREE II standardized scores for domain 4 ranged from $63 \%$ to $89 \%$. Scores of all CPGs were greater than $60 \%$ in domain 4 . This domain was well-addressed in all included CPGs, where recommendations were specific, unambiguous, and easily identifiable in all CPGs. Three CPGs scored more than $80 \%$ (NICE, SMOH, and UMHS).

Domain 5: Applicability. The AGREE II standardized scores for domain 5 ranged from $29 \%$ to $92 \%$. Scores of all CPGs were greater than $60 \%$ in domain 5 except CPGs for CADDRA and NHMRC, where facilitators, barriers, monitoring and auditing criteria, resource implications, and formal cost-analyses were not addressed. NICE CPG received the highest score, being the only guideline that received a score above $90 \%$.

Domain 6: Editorial independence. The AGREE II standardized scores for domain 6 ranged from $28 \%$ to $92 \%$. Scores of all CPGs were greater than $60 \%$ except the SMOH CPG.

Overall assessment. The AGREE II standardized domain scores for overall assessment ranged from $50 \%$ to $100 \%$. All CPGs scored greater than $60 \%$ in the first overall assessment, except AAP, NHMRC and SMOH. Overall the NICE CPG received the highest scores on all six AGREE II domains, in addition to the highest score in the first overall assessment; it was the only CPG that received a score of $100 \%$.

Recommending the CPGs for use in practice. The second (overall) assessment, pertaining to the overall recommendation for using the given CPG in clinical practice, revealed a variation between this score and the individual scores of domains in each CPG. This could be illustrated in the NICE CPG where this second overall assessment did not reflect a similarly high score as the scores received in the other six domains and the first overall assessment (i.e. in it, two assessors recommended NICE CPG for use with modifications, and one recommended it for use without modifications). A similar result was noted in the assessment of the AAP and CADDRA despite lower scores in other domains. UMHS was recommended for use by two appraisers. Nevertheless, there was an observed overall consistency in the recommendations of ADHD management throughout the included CPGs despite the variable strengths and weaknesses in each CPG according to the AGREE II criteria. This included diagnosing ADHD using the DSM-5 criteria, identifying comorbidities, initiation of the psycho-social or psycho-behavioral treatment, different management plans according to the age group, and stepwise approach of the pharmacological treatment with psycho-stimulants as the first-line.

All included CPGs cited systematic reviews and meta-analyses in their references list. The largest number of systematic reviews was observed in the evidence-base of the CPGs from NICE $(\mathrm{N}=67)$, SMOH $(\mathrm{N}=17)$, NHMRC $(\mathrm{N}=14)$, CADDRA $(\mathrm{N}=7), \mathrm{AAP}(\mathrm{N}=2)$, and UMHS $(\mathrm{N}=1)$ in descending order. Cochrane systematic reviews were only included in three CPGs: NICE ( $n=19,28 \%)$, NHMRC $(n=5,36 \%)$, and SMOH $(4,24 \%)$. Moreover, two Cochrane systematic reviews were mentioned in the text of the UMHS CPG but were not cited in the references section and henceforth were considered not reported (0) (S2 Table). Overall, the lines of management of ADHD were similar in these CPGs (S3 Table).

\section{Discussion}

The aim of this systematic review was to explore the quality of and critically appraise recently published evidence-based CPGs for the management of ADHD in all age groups [26]. An 
additional purpose was to assist clinicians and CPG groups in identifying high-quality and trustworthy evidence-based CPGs for ADHD using the AGREE II criteria.

Internationally accepted standards and appraisal tools for evidence-based CPGs recommend the transparent reporting of the CPG development process. This process includes; (i) selection of the health topic, (ii) composition of the CPG development group, (iii) key health questions, (iv) scope of the CPG, (v) systematic evidence review and decision-making process, (vi) formulation and articulation of CPG recommendations, ratings of evidence and recommendations, and evidence-to-recommendations links, (vii) implementation considerations and tools, (viii) peer review and stakeholder consultations, (ix) CPG expiration and updating, (x) financial support and sponsoring organization, and (xi) management of conflicts of interest [14-18]. Our appraisal, conducted using the AGREE II Instrument, highlighted several areas for improvement in the methodological rigor of the ADHD CPGs included for critical appraisal. The ADHD CPGs had several gaps in their Rigor of Development (Domain 3), which is the largest (and the core) AGREE II domain and in their Applicability (Domain 5) as well. Highlighting the importance of these two domains has been suggested [52]. There was consistency in ADHD recommendations despite variable evidence-bases. This consistency may reflect consensus in the healthcare community towards management of ADHD, despite the absence of a strong evidence-base in some CPGs. The AGREE II instrument has undergone several updates and improvements. Some of the shortcomings of the AGREE II instrument has been addressed in a recently developed tool entitled 'AGREE-REX'

(Recommendation EXcellence) that addresses clinical credibility and implementability of the $\mathrm{CPG}$ recommendations. This new tool has been validated and is currently being refined before being shared publicly [53].

To the best of our knowledge, this is the first study to systematically evaluate the quality of recently published CPGs for management of ADHD in all age groups using the complete AGREE II instrument. The ADHD CPG review by the Canadian Agency for Drugs and Technologies in Health, even though limited to pharmacological treatment, is highly consistent with our findings [19]. It listed the AAP, and $\mathrm{SMOH}$ as the most rigorous ADHD CPGs. The aforementioned Alexandria University thesis reviewed 4 ADHD CPGs identified at the time of that study (viz. NICE 2008, AAP 2011, SIGN 2009, and ICSI 2010), with similar findings for the NICE and AAP CPGs as this appraisal [23]. Andrade et al systematically reviewed CPGs for the assessment, prevention and treatment of disruptive behavior (including ADHD, oppositional defiant disorder, conduct disorder and aggression) in children and youth using the AGREE II Instrument. It priotitized three AGREE II domians, viz. domains 2,3, and 6, to classify CPGs [54]. Despite being more broad in the scope of the review in terms of diagnosis (i.e. disruptive behavior) and more specific in terms of the age group (i.e. children and youth), it revealed overall similar results including selecting domain 3(rigor of development) as a key domain for filtering CPGs and displaying NICE as a superior ADHD CPG [54]. Moreover, Andrade chose to use different cutoffs for quality ratings (viz. 50\% for minimum and 70\% for maximum) [54].

Our review showed that only one ADHD CPG applied the GRADE methodology to appraise the quality of evidence (NICE) [31]. The NICE CPG development methodology is based on internationally recognized CPG standards like the AGREE II criteria, the Guideline Implementability Appraisal tool, in addition to primary methodological research and evaluation conducted by NICE. It includes transparent and clear health questions, search strategy, selection criteria for evidence, critical appraisal of clinical and economic evidence, consultation and validation process, and a noted component for implementation considerations and tools [31]. The NHMRC announced on its website that it started developing CPGs using GRADE in 2016 which followed the publication date of its most recent ADHD CPG (2012) [33]. All six CPGs under study included cross-referenced systematic reviews but only three 
CPGs included Cochrane reviews (NICE, NHMRC, and SMOH) despite increased production of Cochrane reviews. Currently, the total number of ADHD-related systematic review protocols registered in the PROSPERO database is 417, which comprises 337 ongoing reviews, 35 completed but not published including this review under study, 39 completed and published, one review is ongoing update, and two reviews that were discontinued [55]. Four Cochrane reviews on; (i) cognitive-behavioral interventions for attention deficit hyperactivity disorder (ADHD) in adults, (ii) methylphenidate for attention deficit hyperactivity disorder (ADHD) in children and adolescents-assessment of adverse events in non-randomised studies; (iii) Pharmacological treatment for attention deficit hyperactivity disorder (ADHD) in children with comorbid tic disorders; and (iv) Amphetamines for attention deficit hyperactivity disorder (ADHD) in adults in addition to 9 protocols were published after the publication of the NICE CPG in $16^{\text {th }}$ of March 2018 [56-59]. Despite the utilization of systematic reviews in the included ADHD CPGs, an area for improvement remains regarding the utilization of highquality systematic reviews like Cochrane reviews in these CPGs. Similar recommendations were reported by Vale et al [60].

Furthermore, other reviews were published for ADHD but none have utilized a validated tool such as the AGREE II Instrument except the review by Siexas et al. that utilized the first version of the AGREE Instrument [61-64]. This appraisal was also conducted by a multidisciplinary team and an expert methodologist, which adds a layer of strength to the assessment.

Moreover, an additional implication for practice is to encourage healthcare providers caring for patients with ADHD to adopt principles of 'Evidence-Based' rather than 'EminenceBased' Healthcare in their daily practice through training and education on CPG standards and appraisal tools [65-68]

One limitation to utilizing the AGREE II instrument is that it does not comprehensively critically appraise other important items included in the GRADE methodology for CPG development (e.g. risk of bias, precision, consistency, directness, and publication bias).The selection of $60 \%$ as a cut-off point for standard domain scores is another potential limitation as the original AGREE II does not mandate such a cut-off but similar studies have used it previously [13]. Other raters may choose different cut-offs [54]

Another limitation of our review was the exclusion of Non-English CPGs from our set of appraised CPGs despite the existence of Dutch, Finnish, Norwegian, German, and Spanish ADHD CPGs. Similar exclusion criteria were selected in published AGREE appraisals for CPGs $[64,69,70]$

The results of this appraisal can be used as a main component of a CPG development or adaptation project for the management of ADHD. Furthermore, it highlights the importance of inclusion of the AGREE II Instrument as a part of the capacity building for clinicians to guide them during the identification and adoption of CPGs for use in their daily practice.

In conclusion, The AGREE II assessment of the six included ADHD CPGs revealed methodological shortcomings in several domains. We recommend several areas for improvement for future CPGs, using the AGREE II criteria and the NICE CPG as a model. This critical appraisal illustrates the importance of regular quality assessment of CPGs by clinicians to ensure the transparency and rigor of the CPG development process and the evidence-base management of patients with ADHD.

\section{Supporting information}

S1 Fig. PRISMA flow diagram. Systematically searching and selecting the clinical practice guidelines for management of ADHD.

(DOC) 


\section{S1 Protocol.}

(PDF)

S1 Table. PRISMA checklist.

(DOC)

S2 Table. Mapping of ADHD CPGs against systematic reviews, meta-analyses and the utilization of the GRADE method.

(DOCX)

S3 Table. Key recommendations of included ADHD CPGs.

(DOCX)

\section{Acknowledgments}

The authors wish to thank Prof. Tarek Omar for sharing the AGREE II scores of ADHD CPGs from the relevant Master thesis from Alexandria University.

The unified ADHD Clinical Practice Guidelines Project is the strategic project 7.2 of the Saudi ADHD Society for the period 2017-2019. The Saudi ADHD Society is a registered nonprofit under license 474 from the Saudi Ministry of Labor and Social Development, and the project received the Ministry approval (No. 52476) on 5/8/1438. The project is entirely funded by the Saudi ADHD Society. No funding was received from any pharmaceutical or industrial company.

gMembers of the Saudi ADHD Society include TA and JLV.

\section{Author Contributions}

Conceptualization: Yasser Sami Amer.

Data curation: Yasser Sami Amer, Jeremy L. Varnham, Fahad A. Bashiri, Muddathir Hamad Hamad, Saleh M. Al Salehi, Hadeel Fakhri Daghash, Turki Homod Albatti.

Formal analysis: Yasser Sami Amer.

Investigation: Yasser Sami Amer, Haya Faisal Al-Joudi, Fahad A. Bashiri, Muddathir Hamad Hamad, Hadeel Fakhri Daghash, Turki Homod Albatti.

Methodology: Yasser Sami Amer.

Project administration: Yasser Sami Amer, Jeremy L. Varnham.

Resources: Yasser Sami Amer, Haya Faisal Al-Joudi, Jeremy L. Varnham, Fahad A. Bashiri, Muddathir Hamad Hamad, Hadeel Fakhri Daghash.

Supervision: Yasser Sami Amer.

Validation: Yasser Sami Amer.

Writing - original draft: Yasser Sami Amer, Haya Faisal Al-Joudi, Jeremy L. Varnham.

Writing - review \& editing: Yasser Sami Amer, Haya Faisal Al-Joudi, Fahad A. Bashiri, Muddathir Hamad Hamad, Saleh M. Al Salehi, Hadeel Fakhri Daghash, Turki Homod Albatti.

\section{References}

1. International Classification of Diseases [Internet]. World Health Organization. 2018 [cited 1 June 2018] http://www.who.int/classifications/icd/en/ 
2. ICD-11-Mortality and Morbidity Statistics [Internet]. Icd.who.int. 2019 [cited 15 March 2019]. https:// icd.who.int/browse11//-m/en\#/ http://id.who.int/icd/entity/821852937

3. DSM-5 [Internet]. Psychiatry.org. 2019 [cited 15 March 2019]. https://www.psychiatry.org/psychiatrists/ practice/dsm

4. Psychiatry Online | DSM Library [Internet]. Dsm.psychiatryonline.org. 2019 [cited 15 March 2019]. https://dsm.psychiatryonline.org/doi/book/10.1176/appi.books.9780890425596

5. Turgay A, Goodman DW, Asherson P, Lasser RA, Babcock TF, Pucci ML et al. Lifespan persistence of ADHD: the life transition model and its application. J Clin Psychiatry. 2012; 73(2):192-201. http://doi. org/10.4088/JCP.10m06628 PMID: 22313720

6. Pliszka SR. Attention-Deficit Hyperactivity Disorder Across the Lifespan. Focus. 2016; 14(1):46-53. https://doi.org/10.1176/appi.focus.20150022

7. American Psychiatric Association. Diagnostic and statistical manual of mental disorders (5th ed.) $\left(D S M-5^{\circledR}\right)$. American Psychiatric Pub, Arlington, VA; 2013 May 22.

8. Biederman J, Faraone SV. Current concepts on the neurobiology of Attention-Deficit/Hyperactivity Disorder. Journal of Attention Disorders. 2002; 6 Suppl 1: S7-16. https://doi.org/10.1177/ 070674370200601 S03

9. Shinwari JM, Al Yemni EA, Alnaemi FM, Abebe D, Al-Abdulaziz BS, Al Mubarak BR, et al. Analysis of shared homozygosity regions in Saudi siblings with attention deficit hyperactivity disorder. Psychiatric genetics. 2017 Aug; 27(4):131. https://doi.org/10.1097/YPG.0000000000000173 PMID: 28452824

10. Sharma A, Couture J. A review of the pathophysiology, etiology, and treatment of attention-deficit hyperactivity disorder (ADHD). Annals of Pharmacotherapy. 2014 Feb; 48(2):209-25. http://doi.org/10. 1177/1060028013510699 PMID: 24259638

11. Biederman J, Newcorn J, Sprich S. Comorbidity of attention deficit hyperactivity disorder with conduct, depressive, anxiety, and other disorders. The American journal of psychiatry. 1991 May 1; 148(5):564. http://doi.org/10.1176/ajp.148.5.564 PMID: 2018156

12. Rabito-Alcon M, Correas-Lauffer J. Treatment guidelines for Attention Deficit and Hyperactivity Disorder: A critical review. Actas Esp Psiquiatr. 2014; 42(6):315-24. PMID: 25388773

13. Sekercioglu N, Al-Khalifah R, Ewusie J, Elias R, Thabane L, Busse J et al. A critical appraisal of chronic kidney disease mineral and bone disorders clinical practice guidelines using the AGREE II instrument. International Urology and Nephrology. 2016; 49(2):273-284. https://doi.org/10.1007/s11255-016-14363 PMID: 27804080

14. Institute of Medicine (US) Committee on Standards for Developing Trustworthy Clinical Practice Guidelines; Graham R, Mancher M, Miller Wolman D, et al., editors. Clinical Practice Guidelines We Can Trust. Washington (DC): National Academies Press (US); 2011. https://www.ncbi.nlm.nih.gov/books/ NBK209539/10.17226/13058

15. Qaseem A, Forland F, Macbeth F, Ollenschläger G, Phillips S, van der Wees $P$ et al. Guidelines International Network: Toward International Standards for Clinical Practice Guidelines. Annals of Internal Medicine. 2012; 156(7):525. https://doi.org/10.7326/0003-4819-156-7-201204030-00009 PMID: 22473437

16. Schünemann HJ, Wiercioch W, Etxeandia I, Falavigna M, Santesso N, Mustafa R, et al. Guidelines 2.0: systematic development of a comprehensive checklist for a successful guideline enterprise. CMAJ Feb 2014, 186 (3) E123-E142. https://doi.org/10.1503/cmaj.131237 PMID: 24344144

17. Brouwers M, Kerkvliet K, Spithoff K. The AGREE Reporting Checklist: a tool to improve reporting of clinical practice guidelines. BMJ. 2016;:;1152. https://doi.org/10.1136/bmj.i1152 PMID: 26957104

18. Amer YS, Elzalabany MM, Omar TI, Ibrahim AG and Dowidar NL. The 'Adapted ADAPTE': an approach to improve utilization of the ADAPTE guideline adaptation resource toolkit in the Alexandria Center for Evidence-Based Clinical Practice Guidelines. J Eval Clin Pract, 2015: 21: 1095-1106. https://doi.org/ 10.1111/jep.12479

19. Vlayen J, Aertgeerts B, Hannes K, Sermeus W, Ramaekers D. A systematic review of appraisal tools for clinical practice guidelines: multiple similarities and one common deficit. J Qual Health Care 2005. 17(3):235-242. https://doi.org/10.1093/intqhc/mzi027 PMID: 15743883

20. Siering U, Eikermann M, Hausner E, Hoffman-Eber W, Neugebauer EA. Appraisal Tools for Clinical Practice Guidelines: a systematic review. PLoS ONE 2013. 8(12); e82915 https://doi.org/10.1371/ journal.pone.0082915 PMID: 24349397

21. Citations of Core Publications-AGREE Enterprise website [Internet]. Agreetrust.org. 2018 [cited 15 June 2019]. https://www.agreetrust.org/resource-centre/citations-of-core-publications/

22. Canadian Agency for Drugs and Technologies in Health (CADTH). Pharmacologic Management of Patients with ADHD: A Review of Guidelines [Internet]. PubMed Health. 2018 [cited 15 June 2019]. https://www.ncbi.nlm.nih.gov/pubmedhealth/PMH0086535/ 
23. Morcos M. Adaptation of international evidence-based clinical practice guidelines for the treatment of attention deficit hyperactivity disorder among children attending Alexandria University Children's Hospital [Master's]. Faculty of Medicine, Alexandria University, Alexandria, Egypt; 2013. http://srv4 eulc.edu.eg/eulc_v5/Libraries/Thesis/BrowseThesisPages.aspx?fn=PublicDrawThesis\&BibID= 11751120

24. Kung J, Miller RR, Mackowiak PA. Failure of Clinical Practice Guidelines to Meet Institute of Medicine Standards: Two More Decades of Little, If Any, Progress. Arch Intern Med. 2012; 172(21):1628-1633. https://doi.org/10.1001/2013.jamainternmed.56 PMID: 23089902

25. Shaneyfelt TM, Mayo-Smith MF, Rothwangl J. Are guidelines following guidelines? the methodological quality of clinical practice guidelines in the peer-reviewed medical literature. JAMA. 1999; 281 (20):1900-190510349893 PMID: 10349893

26. Albatti T, Al Salehi S, Bashiri F, Hamad M, Al-Joudi H, Daghash H, et al. Appraisal of clinical practice guidelines for the management of attention deficit hyperactivity disorder (ADHD) using the AGREE I Instrument: a systematic review. PROSPERO 2017 CRD42017078712 http://www.crd.york.ac.uk/ PROSPERO/display_record.php?ID=CRD42017078712

27. Appraisal of clinical practice guidelines for the management of attention deficit hyperactivity disorder (ADHD) using the AGREE II Instrument: a systematic review v1 (protocols.io.q27dyhn). https://dx.doi. org/10.17504/protocols.io.q27dyhn

28. Fervers B, Burgers J, Voellinger R, Brouwers M, Browman G, Graham I et al. Guideline adaptation: an approach to enhance efficiency in guideline development and improve utilisation. BMJ Quality \& Safety. 2011; 20(3):228-236. https://doi.org/10.1136/bmjqs.2010.043257 PMID: 21209134

29. Altokhais TI, Al-Obaid OA, Kattan AE, Amer YS, CPG Collaborative Groups. Assessment of implementability of an adapted clinical practice guideline for surgical antimicrobial prophylaxis at a tertiary care university hospital. J Eval Clin Pract. 2017; 23:156-164. https://doi.org/10.1111/jep.12658 PMID: 27807920

30. Eady E, Layton A, Sprakel J, Arents B, Fedorowicz Z, van Zuuren E. AGREE II assessments of recent acne treatment guidelines: how well do they reveal trustworthiness as defined by the U.S. Institute of Medicine criteria?. British Journal of Dermatology. 2017; 177(6):1716-1725. https://doi.org/10.1111/ bjd. 15777 PMID: 28667760

31. Developing NICE guidelines: the manual | Guidance and guidelines | NICE [Internet]. Nice.org.uk. 2018 [cited 15 June 2019]. https://www.nice.org.uk/process/pmg20/chapter/introduction-and-overview

32. WHO Handbook for Guideline Development- 2nd Edition [Internet]. Apps.who.int. 2018 [cited 15 June 2019]. http://apps.who.int/medicinedocs/en/d/Js22083en/

33. How NHMRC develops its guidelines | National Health and Medical Research Council [Internet]. Nhmrc.gov.au. 2018 [cited 15 June 2019]. https://www.nhmrc.gov.au/guidelines-publications/hownhmrc-develops-its-guidelines

34. SIGN 50: a guideline developer's handbook [Internet]. Sign.ac.uk. 2018 [cited 15 June 2019]. http:// www.sign.ac.uk/sign-50.html

35. Guyatt G, Oxman A, Vist G, Kunz R, Falck-Ytter Y, Alonso-Coello P et al. GRADE: an emerging consensus on rating quality of evidence and strength of recommendations. BMJ. 2008; 336(7650):924926. https://doi.org/10.1136/bmj.39489.470347.AD PMID: 18436948

36. About GRADE [Internet]. Cebgrade.mcmaster.ca. 2019 [cited 15 June 2019]. https://cebgrade mcmaster.ca/aboutgrade.html

37. ADHD: Clinical Practice Guideline for the Diagnosis, Evaluation, and Treatment of Attention-Deficit/ Hyperactivity Disorder in Children and Adolescents. PEDIATRICS. 2011; 128(5):1007-1022. http:// pediatrics.aappublications.org/content/early/2011/10/14/peds.2011-2654 PMID: 22003063

38. For Health Professionals: Clinical Practice Guidelines: ADHD, University of Michigan Health System [Internet]. Med.umich.edu. 2018 [cited 15 June 2019]. http://www.med.umich.edu/1info/FHP/ practiceguides/adhd.html

39. Canadian ADHD Practice Guidelines | Canadian ADHD Resource Alliance (CADDRA) [Internet]. Caddra.ca. 2018 [cited 15 June 2019]. https://www.caddra.ca/canadian-adhd-practice-guidelines/

40. Clinical Practice Points on the Diagnosis, Assessment and Management of ADHD in Children and Adolescents | National Health and Medical Research Council (NHMRC) [Internet]. Nhmrc.gov.au. 2018 [cited 15 June 2019]. https://www.nhmrc.gov.au/guidelines-publications/mh26

41. ADHD | Ministry of Health [Internet]. Moh.gov.sg. 2014 [cited 15 June 2019]. https://www.moh.gov.sg/ content/moh_web/healthprofessionalsportal/doctors/guidelines/cpg_medical/2014/cpgmed_adhd.html

42. Attention deficit hyperactivity disorder: diagnosis and management / Guidance and guidelines | National Institute for Health and Care Excellence (NICE) [Internet]. Nice.org.uk. March 2018 [cited 15 June 2019]. https://www.nice.org.uk/guidance/cg72 
43. Association of the Scientific Medical Societies in Germany (AWMF) Online. Interdisciplinary Evidenceand Consensus-based Guideline "Attention Deficit/Hyperactivity Disorder in Children, Young People and Adults" [in German] 2018 [cited 15 June 2019]. https://www.awmf.org/uploads/tx_szleitlinien/028045I_S3_ADHS_2018-06.pdf.

44. Working group of the Clinical Practice Guideline on Therapeutic Interventions in Attention Deficit Hyperactivity Disorder (ADHD). Clinical Practice Guideline on Therapeutic Interventions in Attention Deficit Hyperactivity Disorder (ADHD). Ministry of Health, Social Services and Equality. Health Sciences Institute in Aragon (IACS) [in Spanish] 2017 [cited 15 June 2019]. http://www.guiasalud.es/GPC/GPC_574_ TDAH_IACS_compl.pdf.

45. Kooij J, Bijlenga D, Salerno L, Jaeschke R, Bitter I, Balázs J et al. Updated European Consensus Statement on diagnosis and treatment of adult ADHD. European Psychiatry. 2019; 56:14-34. https://doi.org/ 10.1016/j.eurpsy.2018.11.001 PMID: 30453134

46. Bolea-Alamañac B, Nutt D, Adamou M, Asherson P, Bazire S, Coghill D et al. Evidence-based guidelines for the pharmacological management of attention deficit hyperactivity disorder: Update on recommendations from the British Association for Psychopharmacology. Journal of Psychopharmacology. 2014; 28(3):179-203. https://doi.org/10.1177/0269881113519509 PMID: 24526134

47. ADHD Treatment Recommendations / CDC [Internet]. Centers for Disease Control and Prevention. 2019 [cited 15 June 2019]. https://www.cdc.gov/ncbddd/adhd/guidelines.html

48. ADHD Guidelines-ADHD Institute.com [Internet]. ADHD Institute. 2019 [cited 15 June 2019]. https:// adhd-institute.com/disease-management/guidelines/

49. DynaMed Plus [Internet]. Ipswich (MA): EBSCO Information Services. 1995-. Record No. T113926 Attention deficit hyperactivity disorder (ADHD) in children and adolescents; [updated 2018 Nov 30, cited 15 June 2019]. https://www.dynamed.com/topics/dmp AN T113926. Registration and login required.

50. DynaMed Plus [Internet]. Ipswich (MA): EBSCO Information Services. 1995-. Record No. T231898, Attention deficit hyperactivity disorder (ADHD) in adults; [updated 2018 Dec 03, cited 15 June 2019]. https://www. dynamed.com/topics/dmp AN T231898. Registration and login required.

51. The World Federation of ADHD Guide [Internet]. Adhd-federation.org. 2019 [cited 15 June 2019]. https://www.adhd-federation.org/publications/the-word-federation-of-adhd-guide/

52. Anwer MA, Al-Fahed OB, Arif SI, Amer YS, Titi MA, Al-Rukban MO. Quality assessment of recent evidence-based clinical practice guidelines for management of type 2 diabetes mellitus in adults using the AGREE II instrument. J Eval Clin Pract. 2017;1-7. https://doi.org/10.1111/jep.12785

53. Brouwers M, Florez I, McNair S, Vella E, Yao X. Clinical Practice Guidelines: Tools to Support High Quality Patient Care. Seminars in Nuclear Medicine. 2019; 49(2):145-152. https://doi.org/10.1053/j. semnuclmed.2018.11.001 PMID: 30819394

54. Andrade B, Courtney D, Duda S, Aitken M, Craig S, Szatmari P et al. A Systematic Review and Evaluation of Clinical Practice Guidelines for Children and Youth with Disruptive Behavior: Rigor of Development and Recommendations for Use. Clinical Child and Family Psychology Review. 2019; Published online: 29/3/2019. https://doi.org/10.1007/s10567-019-00292-2 PMID: 30927153

55. PROSPERO [Internet]. Crd.york.ac.uk. 2018 [cited 26 June 2019]. NHS National Institute for Health Research. https://www.crd.york.ac.uk/PROSPERO/

56. Lopez P, Torrente F, Ciapponi A, Lischinsky A, Cetkovich-Bakmas M, Rojas J, et al. Cognitive-behavioural interventions for attention deficit hyperactivity disorder (ADHD) in adults. Cochrane Database of Systematic Reviews 2018, Issue 3. Art. No.: CD010840. https://doi.org/10.1002/14651858.CD010840. pub2 PMID: 29566425

57. Storebø OJ, Pedersen N, Ramstad E, Kielsholm ML, Nielsen SS, Krogh HB, et al. Methylphenidate for attention deficit hyperactivity disorder (ADHD) in children and adolescents-assessment of adverse events in non-randomised studies. Cochrane Database of Systematic Reviews 2018, Issue 5. Art. No.: CD012069. https://doi.org/10.1002/14651858.CD012069.pub2

58. Osland ST, Steeves TDL, Pringsheim T. Pharmacological treatment for attention deficit hyperactivity disorder (ADHD) in children with comorbid tic disorders. Cochrane Database of Systematic Reviews 2018, Issue 6. Art. No.: CD007990. https://doi.org/10.1002/14651858.CD007990.pub3 PMID: 29944175

59. Castells X, Blanco-Silvente L, Cunill R. Amphetamines for attention deficit hyperactivity disorder (ADHD) in adults. Cochrane Database of Systematic Reviews 2018, Issue 8. Art. No.: CD007813. https://doi.org/10.1002/14651858.CD007813.pub3 PMID: 30091808

60. Vale C, Rydzewska L, Rovers M, Emberson J, Gueyffier F, Stewart L. Uptake of systematic reviews and meta-analyses based on individual participant data in clinical practice guidelines: descriptive study. BMJ. 2015; 350(mar06 6):h1088-h1088. 
61. Seixas M, Weiss M, Müller U. Systematic review of national and international guidelines on attentiondeficit hyperactivity disorder. Journal of Psychopharmacology. 2012; 26(6):753-765. https://doi.org/10. 1177/0269881111412095 PMID: 21948938

62. Rabito-Alcón María F., and Javier Correas-Lauffer. "Treatment guidelines for attention deficit and hyperactivity disorder: a critical review." Actas Esp Psiquiatr 42.6 (2014): 315-324. PMID: 25388773

63. Banaschewski T, Becker K, Döpfner M, Holtmann M, Rösler M, Romanos M: Attention-deficit/hyperactivity disorder-a current overview. Dtsch Arztebl Int 2017; 114: 149-59. https://doi.org/10.3238/ arztebl.2017.0149

64. Thapar A and Cooper M. Attention deficit hyperactivity disorder. The Lancet 2016; 387(10024): 12401250. https://doi.org/10.1016/S0140-6736(15)00238-X

65. Bhandari M, Zlowodzki M, and Cole PA. From eminence-based practice to evidence-based practice: a paradigm shift. Minnesota Medicine 2004; 87(4): 51-54. PMID: 15144165

66. Ioannidis JPA. Evidence-based medicine has been hijacked: a report to David Sackett. Journal of clinical epidemiology, 2016, 73: 82-86. https://doi.org/10.1016/j.jclinepi.2016.02.012 PMID: 26934549

67. Greenhalgh T, Howick J, and Maskrey N. Evidence based medicine: a movement in crisis?. BMJ 2014; 348: g3725. https://doi.org/10.1136/bmj.g3725 PMID: 24927763

68. Isaacs D and Fitzgerald D. Seven alternatives to evidence based medicine. BMJ, 1999, 319.7225 1618.

69. Bashiri FA, Hamad MH, Amer YS, Abouelkheir MM, Mohamed S, Kentab AY et al. Management of convulsive status epilepticus in children: an adapted clinical practice guideline for pediatricians in Saudi Arabia. Neurosciences. 2017 Apr; 22(2):146. https://doi.org/10.17712/nsj.2017.2.20170093 PMID: 28416791

70. Bazzano AN, Green E, Madison A, Barton A, Gillispie V, Bazzano LA. Assessment of the quality and content of national and international guidelines on hypertensive disorders of pregnancy using the AGREE II instrument. BMJ open. 2016 Jan 1; 6(1):e009189. https://doi.org/10.1136/bmjopen-2015009189 PMID: 26781503 